\title{
Differential microRNA expression profile in the plasma of preeclampsia and normal pregnancies
}

\author{
YAN ZHONG, FUFAN ZHU and YILING DING \\ Department of Gynaecology and Obstetrics, The Second Xiangya Hospital of Central South University, \\ Changsha, Hunan 410011, P.R. China
}

Received April 3, 2016; Accepted March 10, 2017

DOI: $10.3892 /$ etm.2019.7637

\begin{abstract}
Preeclampsia is a common disease in pregnant women that can only be diagnosed from 20 weeks after fertilization. Developing early diagnosis markers is urgent and would be helpful in selecting appropriate treatment strategies. The present study aimed to identify the differential expression profiles of microRNAs in the plasma between patients with preeclampsia and normal pregnancies using microarray methods. Using quantitative polymerase chain reaction (qPCR), the differentially expressed microRNAs (miRNAs or miRs) identified from the microarray analysis were validated. A total of 3 miRNAs, including hsa-miR-1304-5p, hsa-miR-320a and hsa-miR-5002-5p, were upregulated in the plasma of patients with preeclampsia pregnancies. Examination of the functions of these miRNAs demonstrated that they were involved in cell proliferation, indicating that preeclampsia affected this pathway. In addition, 26 downregulated miRNAs were identified by microarray methods. The functions of these miRNAs included immune regulation, vascular development, cancer pathology and pathology of other disease (tuberculosis, oligozoospermia, psoriasis and Alzheimer's disease). Using qPCR, the most differentially expressed miRNAs were confirmed to be hsa-miR-1304-5p, hsa-miR-320a and hsa-miR-5002-5p, which were upregulated, as well as hsa-miR-188-3p, hsa-miR-211-5p, hiv1-miR-TAR-3p, hsa-miR-4432 and hsa-miR-4498 that were significantly downregulated in the plasma of preeclampsia patients. The present findings may be useful in the development of early diagnosis markers and treatment targets for preeclampsia.
\end{abstract}

Correspondence to: Dr Yan Zhong, Department of Gynaecology and Obstetrics, The Second Xiangya Hospital of Central South University, 139 Middle Renmin Road, Changsha, Hunan 410011, P.R. China

E-mail: zhongyan73@csu.edu.cn

Key words: microRNA, microarray, preeclampsia, pregnancies, plasma, diagnosis

\section{Introduction}

Preeclampsia is a common obstetric complication that represents a major factor leading to maternal perinatal mortality and a long-term impact on maternal and child health (1). Preeclampsia in pregnant women results in high risk of cerebrovascular accident, placental abruption and postpartum hemorrhage, while higher risks of fetal distress, stillbirth and neonatal asphyxia have been observed in the fetus (2). In addition, the risk of cerebral infarction and diabetes increases by 2-8 times within 20 years in preeclampsia patients (3). To date, the effect of conventional treatments on preeclampsia is not satisfactory. One of the reasons is that the intervention is always performed during the late pregnancy stages. However, once the placental pathology is formed, it is difficult for healing to occur $(4,5)$. Thus, it is important to develop early diagnosis markers and perform early intervention in preeclampsia patients.

MicroRNA (miRNA or miR) is a small endogenous non-coding RNA molecule, which is one of the most important regulators of gene expression of protein-coding genes, serving an important role in post-transcriptional regulation $(6,7)$. High expression levels of multiple miRNAs in the placenta have been demonstrated to regulate cell proliferation, apoptosis, migration and invasion (8). Furthermore, several miRNAs were specifically expressed in the placenta and released into the maternal blood via exocytosis (9). Comparative analysis in cases of normal pregnancy and preeclampsia patients identified abnormal miRNA expression in preeclampsia placenta. For instance, miRNA-210 is upregulated in preeclampsia placentas, and inhibits the expression levels of homeobox A9 and ephrin A3, leading to reduced cell migration and invasion (10). miR-17-92 and its paralogous miRNA-106a-363 were downregulated in the cell differentiation of trophoblasts, and their overexpression inhibited the cell differentiation (11). Previous studies have also reported the differential expression of miRNAs in the placenta at different stages of pregnancy $(12,13)$. At the early stages of pregnancy, miRNAs that participate in tumor-derived, angiogenic, anti-apoptotic processes are highly expressed in the placenta; by contrast, miRNAs that mediate cell differentiation, tumor progression and immune regulation are highly expressed in the placenta at later stages of pregnancy (14). However, to the best of our knowledge, no previous studies have investigated the 
expression profiles of miRNAs in the plasma of patients with preeclampsia pregnancies. This information may assist in the development of novel diagnosis markers.

Microarray analysis as a method to identify differential expression is a cheap and high efficient approach. In a previous study, using microarray, differentially expressed miRNAs were identified in the placenta of patients with preeclampsia and normal pregnancies (15). The present study aimed to investigate the differential expression of miRNAs in the plasma of preeclampsia and normal pregnancies. The findings of the study may be helpful for developing novel methods for the diagnosis of preeclampsia pregnancies at an early stage.

\section{Materials and methods}

Patients and sampling. Plasma was collected from women $(26.83 \pm 1.17$ years old) from April to May 2014 with preeclampsia pregnancies $(n=3)$ and normal pregnancies $(n=3)$ at the Second Xiangya Hospital of Central South University (Changsha, China). The plasma levels of placental protein 13 (PP13), placental growth factor (PlGF), soluble fms-like tyrosine kinase-1 (sFlt-1), pregnancy-associated plasma protein A (PAPP-A) and human chorionic gonadotropin (hCG) were determined using an AutoDELFIA immunoassay analyzer (Xi'an Yima Opto-electrical Technology Com., Ltd, Shaanxi, China), according to a previously published protocol (16). Patients with preeclampsia presented typical characteristics, according to the diagnostic criteria of preeclampsia (17). All the plasma samples were collected at the 11 weeks after fertilization without any prior clinical treatment to investigate early diagnostic index, while diagnosis was only confirmed at 20 weeks of pregnancy. Informed consents were obtained from the preeclampsia and normal pregnancy individuals. The present study was approved by the Research Ethics Committee of Second Xiangya Hospital of Central South University.

RNA isolation and analysis. The total RNAs were extracted from the plasma using RNA TRIzol reagent (Invitrogen; Thermo Fisher Scientific, Inc., Waltham, MA, USA) according to the manufacturer's instructions. Agarose gel electrophoresis (1.2\%) was used to detected RNA integrity. The quality and quantity of the total RNAs were then analyzed using Agilent 2100 bioanalyzer (Agilent Technologies, Inc., Santa Clara, CA, USA). Only samples with an RNA integrity number (RIN) of $>7.0$ were used in subsequent analyses.

Microarray analysis. Total RNA samples from the preeclampsia pregnancies $(n=3)$ were mixed to obtain the preeclampsia pregnancy group, while samples from normal pregnancies $(\mathrm{n}=3)$. The RNA groups $(1 \mu \mathrm{g}$ each) were examined by Human miRNA Expression Microarray Release 14.0 (Agilent Technologies, Inc.). The experiment and quality control were performed by CapitalBio Corp. (Beijing, China) following the manufacturer's instructions. Subsequently, the signals of the hybridization were obtained and analyzed using an Agilent G2565CA Microarray Scanner system (Agilent Technologies, Inc.). Differential expression of miRNAs between the preeclampsia and normal pregnancies was determined when P-values were $<0.05$. Using the Agilent
GeneSpring software (version 7.3; Agilent Technologies, Inc.), the fold-changes of miRNAs were calculated.

Quantitative polymerase chain reaction ( $q P C R)$. Using qPCR, the miRNA expression profiles of the microarray analysis results were validated. cDNA was synthesized from $1 \mu \mathrm{g}$ total RNA and AMV Reverse Transcriptase kit (Takara, Dalian, China). Next, qPCR analysis was performed on an ABI PRISM 7500 system (Applied Biosystems; Thermo Fisher Scientific, Inc.). The thermal cycling conditions of the reaction were as follows: $95^{\circ} \mathrm{C}$ for $5 \mathrm{~min}$, followed by 40 cycles of $10 \mathrm{sec}$ at $95^{\circ} \mathrm{C}$ and extension for $30 \mathrm{sec}$ at $60^{\circ} \mathrm{C}$. qPCR was performed using the miScript SYBR-Green PCR kit (Qiagen, Hilden, Germany) according to the manufacturer's instructions. The primers (hsa-miR-1304-5p, cat. no. MS00031395; hsa-miR-320a, cat. no. MS00014707; hsa-miR-188-3p, cat. no. MS00008897; hsa-miR-211-5p, cat. no. MS00003808; hiv1-miR-TAR-3p, cat. no. MS00015393; hsa-miR-4432, cat. no. MS00041363; hsa-miR-4498, cat. no. MS00045003; hsa-miR-5002-5p, cat. no. MS00038857) were purchased from Qiagen. The expression levels of miRNAs were calculated according to the $2^{-\Delta \Delta \mathrm{Cq}}$ method (18) and normalized to the U6 expression.

Function analysis of miRNAs. The function of the differentially expressed miRNAs was defined using the Targetscan software (www.targetscan.org) and the miRDB database (http://mirdb.org/miRDB/).

Statistical analysis. Significance differences between the two groups were analyzed using a student's t-test. The data are represented as the mean \pm standard error. A value of $\mathrm{P}<0.05$ was confirmed to indicate a statistically significant difference. All the statistical analyses were conducted using SPSS version 13.0 (SPSS, Inc., Chicago, IL, USA).

\section{Results}

Clinical characteristics of preeclampsia and normal pregnancies. The levels of various factors in the plasma of women with preeclampsia pregnancies are shown in Table I. Certain of these characteristics were significantly different in preeclampsia compared with the values in normal pregnancies. More specifically, PIGF was significantly lower in the preeclampsia patients compared with the control group, while hCG and PP13 were significantly increased in the preeclampsia group. However, sFlt-1 and PAPP-A levels were not markedly different in the plasma between the control and preeclampsia groups.

Quality control of RNAs. The quality of the total RNA in each plasma sample was determined using an Agilent 2100 bioanalyzer, and the RIN values of samples were found to be $>7.0$; 9.3 for patients with preeclampsia and 8.7 for control patients (Fig. 1A). The $18 \mathrm{~S}$ and $28 \mathrm{~S}$ ribosomal RNA (rRNA) peaks were clearly high, and the $5 \mathrm{~S}$ rRNA peak was hardly observed. Agarose gel electrophoresis also demonstrated clear 28S and $18 \mathrm{~S}$ peaks, while the $5 \mathrm{~S}$ peak was hardly observed (Fig. 1B). Thus, the quality and quantity of these total RNAs were in accordance with the requirements for performing microarray analysis. 
Table I. Characteristics of women with preeclampsia and normal pregnancies included in the present study.

\begin{tabular}{lcc}
\hline Parameter & Control group $(\mathrm{n}=3)$ & Preeclampsia patients $(\mathrm{n}=3)$ \\
\hline sFlt-1 $(\mathrm{pg} / \mathrm{ml})$ & $1,427.84 \pm 465.44$ & $1,579.45 \pm 478.96$ \\
PlGF $(\mathrm{pg} / \mathrm{ml})$ & $49.52 \pm 5.81$ & $38.21 \pm 6.78^{\mathrm{a}}$ \\
PAPP-A $(\mathrm{mU} / \mathrm{l})$ & $5,549.33 \pm 431.47$ & $4,732.74 \pm 786.48$ \\
$\mathrm{hCG}(\mathrm{mIU} / \mathrm{ml})$ & $23,736.33 \pm 67.43$ & $61,250.67 \pm 4,127.69^{\mathrm{a}}$ \\
PP13 $(\mathrm{pg} / \mathrm{ml})$ & $433.12 \pm 37.77$ & $580.08 \pm 43.72^{\mathrm{a}}$ \\
\hline
\end{tabular}

${ }^{\mathrm{a}} \mathrm{P}<0.05$ vs. control group. sFlt-1, soluble fms-like tyrosine kinase-1; PlGF, placental growth factor; PAPP-A, pregnancy-associated plasma protein A; hCG, human chorionic gonadotropin; PP13, placental protein 13.

A

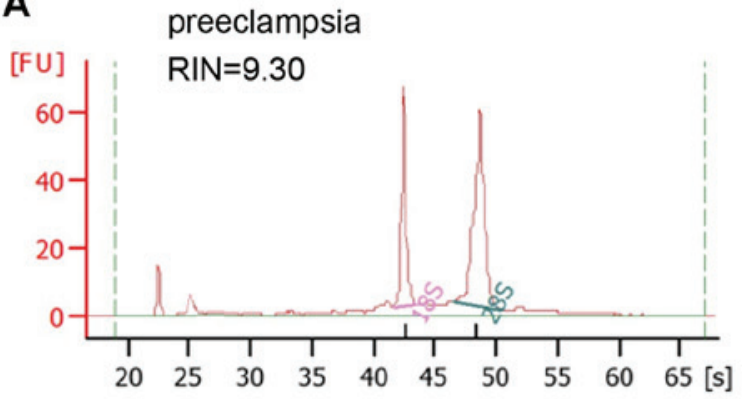

control

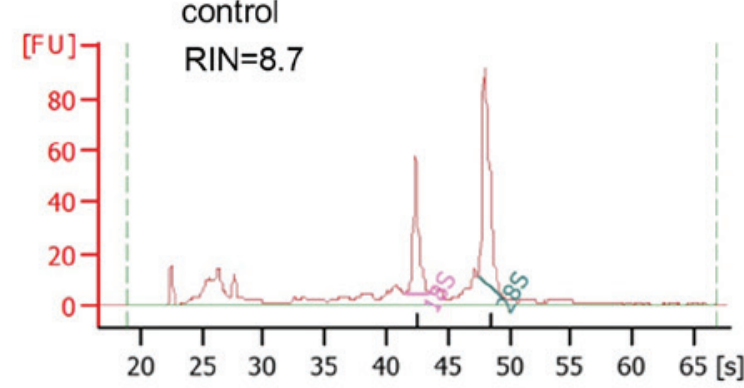

B

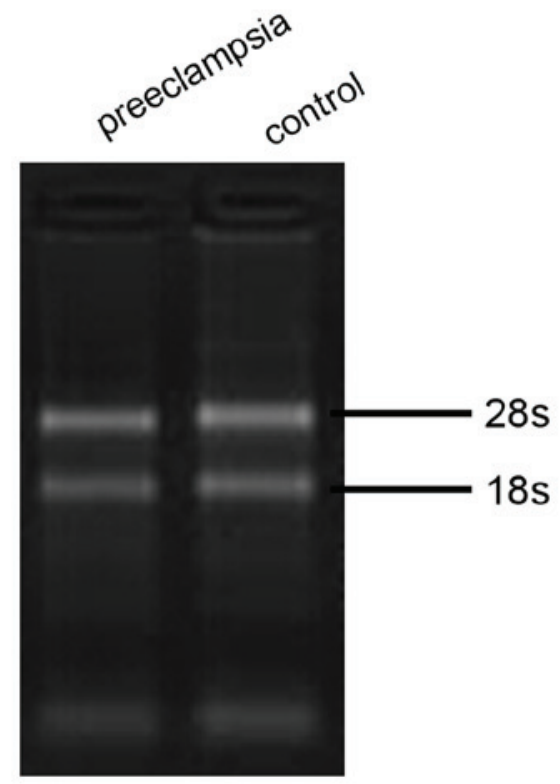

Figure 1. Quality control of the mixed total RNAs of three individuals in each group. (A) RNA quality analysis by Agilent 2100 Bioanalyzer, and (B) agarose gel analysis of total RNA obtained from the plasma of women with preeclampsia and normal pregnancies.

Differential expression of miRNAs in the plasma of preeclampsia and normal pregnancies. Microarray analysis demonstrated that 3 miRNAs were upregulated and 26 miRNAs were downregulated in the plasma of women with preeclampsia pregnancies, when compared with their expression in normal pregnancies (Fig. 2; Table II). The upregulated miRNAs were hsa-miR-1304-5p (2.10-fold), hsa-miR-320a (2.26-fold) and hsa-miR-5002-5p (1.63-fold). The fold-change of downregulated miRNAs was between 0.26 and 0.66 , and the 5 most downregulated miRNAs were hsa-miR-188-3p, hsa-miR-211-5p, hiv1-miR-TAR-3p, hsa-miR-4432 and hsa-miR-4498. These results identified by microarray were then confirmed by qPCR analysis. The results of qPCR were similar to the microarray findings, indicating that hsa-miR-1304-5p, hsa-miR-320a and hsa-miR-5002-5p had significantly higher expression levels in the plasma of preeclampsia pregnancy patients (Fig. 3A). Furthermore, hsa-miR-188-3p, hsa-miR-211-5p, hiv1-miR-TAR-3p, 
Table II. Differential expression of miRNAs between preeclampsia and normal pregnancies.

A, Upregulated in preeclampsia

\begin{tabular}{lcc}
\hline miRNA & Fold-change & P-value \\
\hline hsa-miR-1304-5p & 2.10 & 0.05 \\
hsa-miR-320a & 2.25 & 0.05 \\
hsa-miR-5002-5p & 1.63 & 0.04 \\
\hline
\end{tabular}

$\mathrm{B}$, Downregulated in preeclampsia

\begin{tabular}{llr}
\hline miRNA & Fold-change & P-value \\
\hline hsa-miR-188-3p & 0.26 & $<0.01$ \\
hsa-miR-211-5p & 0.32 & 0.05 \\
hiv1-miR-TAR-3p & 0.33 & $<0.01$ \\
hsa-miR-4498 & 0.40 & 0.03 \\
hsa-miR-4432 & 0.40 & 0.05 \\
hsa-miR-3184-5p & 0.42 & $<0.01$ \\
hsa-miR-92a-2-5p & 0.45 & 0.05 \\
hsa-miR-424-3p & 0.45 & $<0.01$ \\
hsa-miR-5582-3p & 0.47 & 0.05 \\
hsa-miR-1273c & 0.47 & 0.03 \\
hsa-miR-3171 & 0.5 & 0.04 \\
hsa-miR-203a-3p & 0.51 & 0.04 \\
ebv-miR-BART1-5p & 0.53 & $<0.01$ \\
hsa-miR-5009-3p & 0.53 & 0.04 \\
hsa-miR-892b & 0.54 & 0.04 \\
hsa-miR-5000-5p & 0.57 & 0.02 \\
hsa-miR-107 & 0.58 & 0.03 \\
hsa-miR-3649 & 0.6 & $<0.01$ \\
hsa-miR-4482-3p & 0.6 & 0.03 \\
hsa-miR-506-5p & 0.62 & 0.01 \\
hsa-miR-2392 & 0.62 \\
hsa-miR-642b-3p & 0.64 & 0.04 \\
hsa-miR-4758-5p & 0.64 \\
hsa-miR-369-3p & 0.65 \\
hsa-miR-4329 & & 0.02 \\
hsa-miR-3064-5p & & \\
\hline
\end{tabular}

The fold-change was calculated by Agilent GeneSpring software. miR or miRNA, microRNA.

hsa-miR-4432 and hsa-miR-4498 demonstrated significantly lower expression in preeclampsia compared with normal pregnancies $(\mathrm{P}<0.05$; Fig. 3B).

Functional analysis of differentially expressed miRNA. The upregulated miRNAs in preeclampsia pregnancies, including hsa-miR-1304-5p, hsa-miR-320a and hsa-miR-5002-5p, were involved in the cell proliferation function. Furthermore, the 26 downregulated miRNAs in preeclampsia pregnancies were assigned into several functional categories, including immune regulation, vascular development, cancer pathology

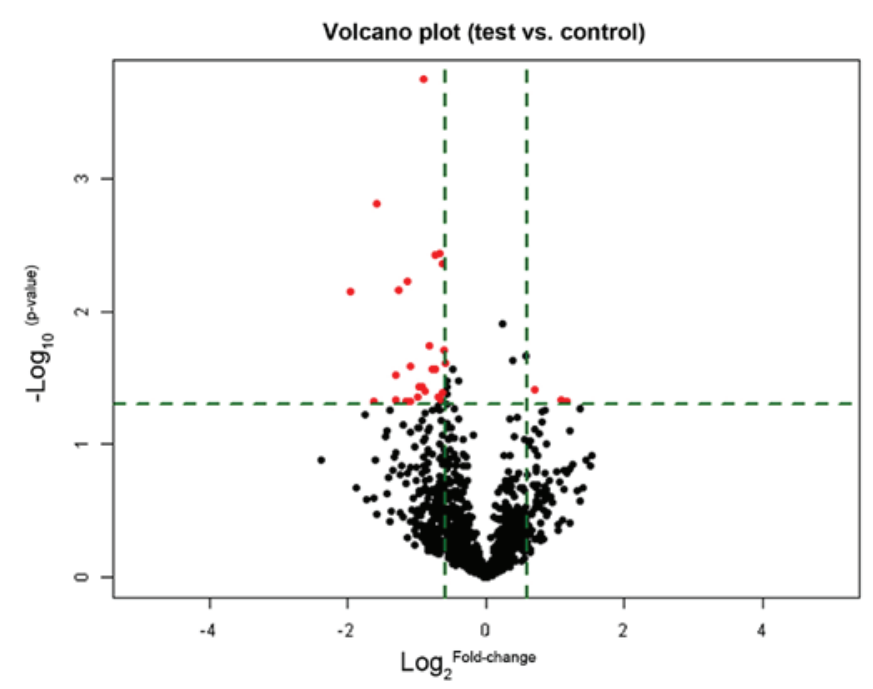

Figure 2. Differences in miRNA expression in the plasma between the preeclampsia and normal pregnancies are shown by a volcano plot. Red dots represent the significantly differentially expressed miRNAs.

and pathology of other disease (tuberculosis, oligozoospermia, psoriasis and Alzheimer's disease). The functions of these miRNAs are shown in Table III.

\section{Discussion}

Preeclampsia is a common disease in human pregnancy, and its characteristics include elevation of blood pressure after 20 weeks of pregnancy and high expression of protein markers in urine $(19,20)$. The symptoms in pregnant women contain headaches, abdominal pain and visual impairment $(21,22)$. In addition, the miscarriage and stillbirth risks are high in preeclampsia patients (23). Due to the late diagnosis of preeclampsia (20 weeks), the efficacy of treatment is poor. Developing novel markers for earlier diagnosis is urgent in order to establish new treatment strategies. In the present study, the miRNAs that were differentially expressed in the plasma of preeclampsia patients were identified by microarray chip analysis. The candidate differentially expressed miRNAs are potential markers for early diagnosis of preeclampsia. In addition, these miRNAs may be new therapeutic target for the treatment of preeclampsia in the future.

Among all the miRNAs identified from the microarray analysis, only 3 miRNAs were significantly upregulated in the plasma of preeclampsia patients. These miRNAs had cell proliferation functions. hsa-miR-1304-5p has previously been demonstrated to be associated with human non-small cell lung cancer cell growth (24). Furthermore, hsa-miR-320a has been shown to mediate cell proliferation of colon cancer cells via targeting $\beta$-catenin (25). Although the evidence on hsa-miR-5002-5p is currently limited, a previous study revealed that it participates in tumorigenesis and cancer cell proliferation (26). Thus, based on results of the current study, cell proliferation-associated miRNAs were upregulated significantly in preeclampsia. Preeclampsia presents with high blood pressure, while it also has characteristics of cell proliferation (27). The present results provided a 
A
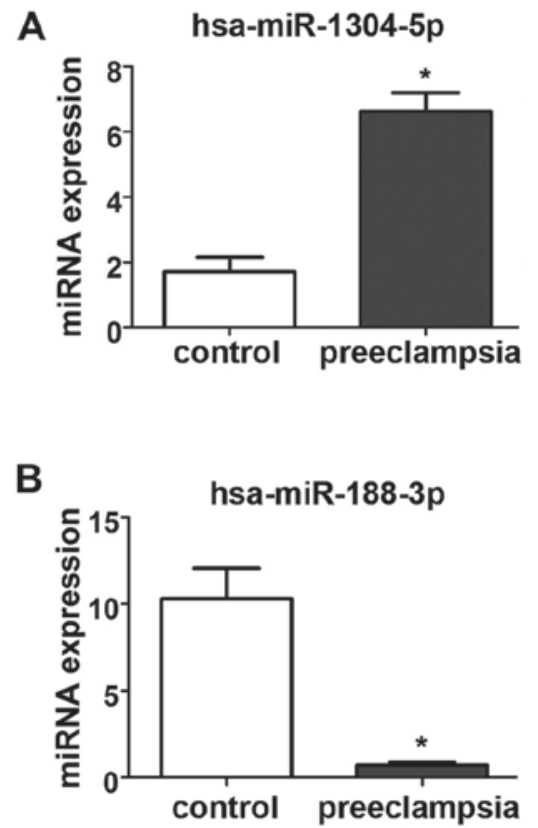

hsa-miR-320a

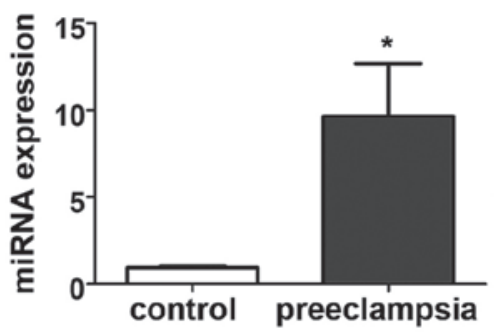

hsa-miR-211-5p

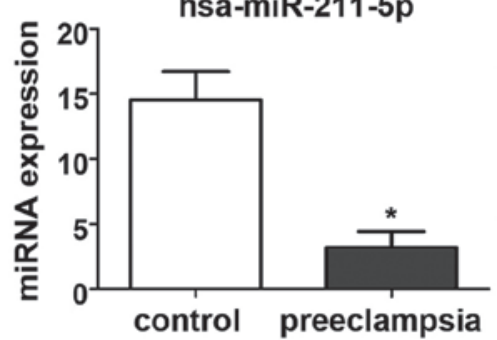

hsa-miR-5002-5p
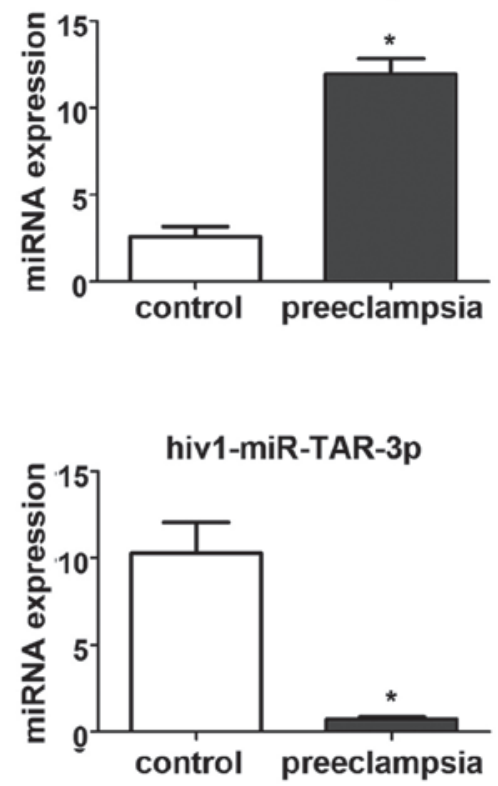
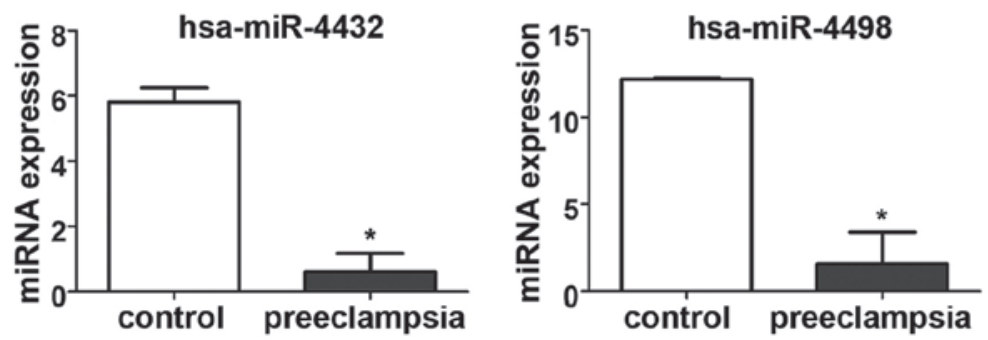

Figure 3. qPCR results demonstrating the significant differences in miRNA expression in the plasma between the preeclampsia and normal pregnancies (A) The 3 upregulated and (B) 5 most downregulated miRNAs in preeclampsia pregnancies were analyzed by qPCR. "P<0.05 vs. normal pregnancy (control) group. qPCR, quantitative polymerase chain reaction.

molecular explanation for this phenomenon. At the early stage of preeclampsia, rapid growth of cells guarantees the requirements for embryos and placenta (28). In the present study, the highly expressed miRNAs suggested abnormal cell proliferation in preeclampsia, and these miRNAs may be novel markers for early diagnosis of preeclampsia.

Among all the downregulated miRNAs in the plasma of preeclampsia patients, the main functional category was immune function. The miRNAs with the lowest expression, including hsa-miR-188-3p, hsa-miR-211-5p, hiv1-miR-TAR-3p, hsa-miR-4432 and hsa-miR-4498, were demonstrated to participate in immune regulation. For instance, hsa-miR-188-3p participates in the growth of $\mathrm{T}$ cells (29), hsa-miR-211-5p is dysregulated in the plasma after hepatitis $\mathrm{C}$ virus infection (30), and hiv1-miR-TAR-3p expression is altered following human immunodeficiency virus-1 infection (31). Furthermore, hsa-miR-4432 is associated with the pathogenesis of chronic lymphocytic leukemia, which also represents immune dysfunction (32). Alteration of hsa-miR-4498 was also observed in major depressive disorder, which is associated with immune dysfunction (33). Based on these previous observations, the downregulation of several immune function-associated miRNAs in the plasma of preeclampsia patients indicated a depressive effect of preeclampsia on the immune system. In addition, the present study demonstrated that certain miRNAs associated with cancer pathology were downregulated in preeclampsia, and these miRNAs may suppress the pathway of cell migration and cell cycle progression. Finally, other miRNAs were identified that participated in several diseases, including tuberculosis (34), oligozoospermia (35), psoriasis (36) and Alzheimer's disease (37). These results revealed that the abnormal expression of the miRNAs may lead to the disease pathology.

In conclusion, the present study identified the differentially expressed miRNAs between preeclampsia and normal pregnancies in the plasma of patients by microarray analysis. The upregulated miRNAs in preeclampsia pregnancies participated in cell proliferation, while the downregulated miRNAs mainly participated in immune regulation, cancer pathology and the pathology of other disease. The significant difference in the expression of these miRNAs was further confirmed by qPCR. The differentially expressed miRNAs in the plasma of preeclampsia and normal pregnancies may serve as novel early diagnostic markers and treatment targets.

\section{Acknowledgements}

Not applicable. 
Table III. Function of miRNAs with differential expression between preeclampsia.

A, Upregulated in preeclampsia

miRNA Functions

hsa-miR-1304-5p

hsa-miR-320a

hsa-miR-5002-5p
Associated with human non-small cell lung cancer cell growth Mediate cell proliferation of colon cancer cells

Tumorigenesis and cancer cell proliferation

$\mathrm{B}$, Downregulated in preeclampsia

miRNA

hsa-miR-188-3p

hsa-miR-211-5p

hiv1-miR-TAR-3p

hsa-miR-4498

hsa-miR-4432

hsa-miR-3184-5p

hsa-miR-92a-2-5p

hsa-miR-424-3p

hsa-miR-5582-3p

hsa-miR-1273c

hsa-miR-3171

hsa-miR-203a-3p

ebv-miR-BART1-5p

hsa-miR-5009-3p

hsa-miR-892b

hsa-miR-5000-5p

hsa-miR-107

hsa-miR-3649

hsa-miR-4482-3p

hsa-miR-506-5p

hsa-miR-2392

hsa-miR-642b-3p

hsa-miR-4758-5p

hsa-miR-369-3p

hsa-miR-4329

hsa-miR-3064-5p

hsa-miR-188-3p
Functions

Growth of T cells

Associated with Hepatitis $\mathrm{C}$ virus infection

Associated with human immunodeficiency virus-1 infection

Associated with immune dysfunction

Pathogenesis of chronic lymphocytic leukemia

Associated with breast cancer

Associated with nasopharyngeal carcinoma metastasis

Associated with lung cancer metastasis

Associated with differentiated thyroid carcinoma

Associated with gastric cancer

Associated with glioblastoma

Associated with epstein-Barr virus infection

Associated with nasopharyngeal carcinoma

Associated with Colon cancer

Pathogenesis of glioblastoma multiforme

Pathogenesis of Alzheimer's disease

Associated with human non-small cell lung cancer

Pathogenesis of glioblastoma

Pathogenesis of thrombocytopenia

Microvascular proliferation in glioblastoma

Pathogenesis of colon cancer

Associated with breast cancer

Associated with pancreatic cancer

Associated with psoriasis

Associated with tuberculosis

Associated with ovarian cancer

Growth of T cells

miR or miRNA, microRNA.

\section{Funding}

This study was supported by grants from the National Natural Science Foundation of China (grant no. 81300503) and PhD Programs Foundation of Ministry of Education of China (grant no. 20130162120062).

\section{Availability of data and materials}

All the data in the current article are available from the authors on request.

\section{Authors' contributions}

YZ and YD participated in project conception and in the study design. FZ and YZ analyzed the data and corrected the manuscript. YD wrote the manuscript. All authors have read and approved the final manuscript.

\section{Ethics approval and consent to participate}

Informed consents were obtained from the preeclampsia and normal pregnancy individuals. The present study was approved 
by the Research Ethics Committee of Second Xiangya Hospital of Central South University (Changsha, China).

\section{Consent for publication}

All the patients provided written informed consent for the publication. The associated data and accompanying images were approved to publish by the patients.

\section{Competing interests}

The authors declare that they have no competing interests.

\section{References}

1. Roberts JM, Taylor RN, Musci TJ, Rodgers GM, Hubel CA and McLaughlin MK: Preeclampsia: An endothelial cell disorder. Am J Obstet Gynecol 161: 1200-1204, 1989.

2. Norwitz ER, Hsu CD and Repke JT: Acute complications of preeclampsia. Clin Obstet Gynecol 45: 308-329, 2002.

3. Ray JG, Vermeulen MJ, Schull MJ and Redelmeier DA: Cardiovascular health after maternal placental syndromes (CHAMPS): Population-based retrospective cohort study. Lancet 366: 1797-1803, 2005 .

4. Zhong Y, Tuuli M and Odibo AO: First-trimester assessment of placenta function and the prediction of preeclampsia and intrauterine growth restriction. Prenat Diagn 30: 293-308, 2010.

5. Steegers EA, von Dadelszen P, Duvekot JJ and Pijnenborg R: Pre-eclampsia. Lancet 376: 631-644, 2010.

6. Bartel DP: MicroRNAs: Genomics, biogenesis, mechanism, and function. Cell 116: 281-297, 2004

7. Bartel DP: MicroRNAs: Target recognition and regulatory functions. Cell 136: 215-233, 2009.

8. Wienholds E and Plasterk RH: MicroRNA function in animal development. FEBS Lett 579: 5911-5922, 2005.

9. Chiu RW and Lo YD: Pregnancy-associated microRNAs in maternal plasma: A channel for fetal-maternal communication? Clin Chem 56: 1656-1657, 2010.

10. Zhang Y, Fei M, Xue G, Zhou Q, Jia Y, Li L, Xin H and Sun S: Elevated levels of hypoxia-inducible microRNA-210 in pre-eclampsia: New insights into molecular mechanisms for the disease. J Cell Mol Med 16: 249-259, 2012.

11. Kumar P, Luo Y, Tudela C, Alexander JM and Mendelson CR The c-Myc-regulated microRNA-17 92 (miR-17 92) and miR-106a 363 clusters target hCYP19A1 and hGCM1 to inhibit human trophoblast differentiation. Mol Cell Biol 33: 1782-1796, 2013.

12. Chim SS, Shing TK, Hung EC, Leung TY, Lau TK, Chiu RW and Lo YM: Detection and characterization of placental microRNAs in maternal plasma. Clin Chem 54: 482-490, 2008.

13. Luo SS, Ishibashi O, Ishikawa G, Ishikawa T, Katayama A, Mishima T, Takizawa T, Shigihara T, Goto T, Izumi A, et al: Human villous trophoblasts express and secrete placenta-specific microRNAs into maternal circulation via exosomes. Biol Reprod 81: 717-729, 2009.

14. Gu Y, Sun J, Groome LJ and Wang Y: Differential miRNA expression profiles between the first and third trimester human placentas. Am J Physiol Endocrinol Metab 304: E836-E843, 2013.

15. Zhu X, Li Y and Jiang F: Differential expression and its clinical significance of miRNA in placentas from women with preeclampsia and normal pregnancy. Modern Med Health 1: 3-4, 2011 (In Chinese).

16. Le Jie: Obstetrics and Gynecology. People's Medical Publishing House, Beijing, pp92-101, 2008.

17. Di Lorenzo G, Ceccarello M, Cecotti V, Ronfani L, Monasta L, Vecchi Brumatti L, Montico M and D'Ottavio G: First trimester maternal serum PIGF, free $\beta$-hcg, PAPP-A, PP-13, uterine artery doppler and maternal history for the prediction of preeclampsia. Placenta 33: 495-501, 2012.

18. Livak KJ, and Schmittgen TD: Analysis of relative gene expression data using real-time quantitative PCR and the 2(-Delta Delta C(T))method. Methods 25: 402-408, 2001.

19. Sibai BM: Diagnosis and management of gestational hypertension and preeclampsia. Obstet Gynecol 102: 181-192, 2003.
20. Wagner LK: Diagnosis and management of preeclampsia. Am Fam Physician 70: 2317-2324, 2004.

21. Jonsson Y, Rubèr M, Matthiesen L, Berg G, Nieminen K, Sharma S, Ernerudh J and Ekerfelt C: Cytokine mapping of sera from women with preeclampsia and normal pregnancies. J Reprod Immunol 70: 83-91, 2006.

22. Suarez B, Alves K, Senat MV, Fromageot J, Fischer C, Rosenberg P and Ville Y: Abdominal pain and preeclampsia: Sonographic findings in the maternal liver. J Ultrasound Med 21: 1077-1083, 2002.

23. Pabinger I, Grafenhofer H, Kaider A, Ilic A, Eichinger S, Quehenberger P, Husslein P, Mannhalter C and Lechner k: Preeclampsia and fetal loss in women with a history of venous thromboembolism. Arterioscler Thromb Vasc Biol 21: 874-879, 2001.

24. Li CG, Pu MF, Li CZ, Gao M, Liu MX, Yu CZ, Yan H, Peng C, Zhao Y, Li Y, et al: microRNA-1304 suppresses human non-small cell lung cancer cell growth in vitro by targeting heme oxygenase-1. Acta Pharmacol Sin 38: 110-119, 2017.

25. Sun JY, Huang Y, Li JP, Zhang X, Wang L, Meng YL, Yan B, Bian YQ, Zhao J, Wang WZ, et al: MicroRNA-320a suppresses human colon cancer cell proliferation by directly targeting $\beta$-catenin. Biochem Biophys Res Commun 420: 787-792, 2012.

26. Berillo O, Regnier M and Ivashchenko A: Binding of intronic miRNAs to the mRNAs of host genes encoding intronic miRNAs and proteins that participate in tumourigenesis. Comput Biol Med 43: 1374-1381, 2013.

27. Wang A, Rana S and Karumanchi SA: Preeclampsia: The role of angiogenic factors in its pathogenesis. Physiology (Bethesda) 24: $147-158,2009$.

28. Li J, Ying H, Cai G, Guo Q and Chen L: Pre-eclampsia-associated reduction in placental growth factor impaired beta cell proliferation through PI3k signalling. Cell Physiol Biochem 36: 34-43, 2015.

29. Hewagama A, Gorelik G, Patel D, Liyanarachchi P, McCune WJ, Somers E, Gonzalez-Rivera T; Michigan Lupus Cohort, Strickland $\mathrm{F}$ and Richardson B: Overexpression of X-linked genes in T cells from women with lupus. J Autoimmun 41: 60-71, 2013.

30. Zhou Y, Sun L, Wang X, Zhou L, Li J, Liu M, Wang F, Peng J, Gui $\mathrm{X}$, Zhao $\mathrm{H}$, et al: Heroin use promotes $\mathrm{HCV}$ infection and dysregulates HCV-related circulating microRNAs. J Neuroimmune Pharmacol 10: 102-110, 2015.

31. Ouellet DL, Vigneault-Edwards J, Létourneau K, Gobeil LA, Plante I, Burnett JC, Rossi JJ and Provost P: Regulation of host gene expression by HIV-1 TAR microRNAs. Retrovirology 10: 86, 2013.

32. Ghanbari M, Sedaghat S, de Looper HW, Hofman A, Erkeland SJ, Franco OH and Dehghan A: The association of common polymorphisms in miR-196a2 with waist to hip ratio and miR-1908 with serum lipid and glucose. Obesity (Silver Spring) 23: 495-503, 2015.

33. Qiao-li Z, Jim L, Xin-yang S, Wei G, Lin Z, Hui-min F, Ai-fang Z, Wei N, Yun-hua D, Li-yi Z, et al: A preliminary analysis of association between plasma microRNA expression alteration and symptomatology improvement in major depressive disorder (MDD) patients before and after antidepressant treatment. Eur J Psychiatry 28: 252-264, 2014.

34. Fu Y, Yi Z, Wu X, Li J and Xu F: Circulating microRNAs in patients with active pulmonary tuberculosis. J Clin Microbiol 49: 4246-4251, 2011

35. Xu M, Qin Y, Qu J, Lu C, Wang Y, Wu W, Song L, Wang S, Chen F, Shen $\mathrm{H}$, et al: Evaluation of five candidate genes from GWAS for association with oligozoospermia in a Han Chinese population. PLoS One 8: e80374, 2013.

36. Guo S, Zhang W, Wei C, Wang L, Zhu G, Shi Q, Li S, Ge R, Li K, Gao L, et al: Serum and skin levels of miR-369-3p in patients with psoriasis and their correlation with disease severity. Eur J Dermatol 23: 608-613, 2013.

37. Wang WX, Rajeev BW, Stromberg AJ, Ren N, Tang G, Huang Q, Rigoutsos I and Nelson PT: The expression of microRNA miR-107 decreases early in Alzheimer's disease and may accelerate disease progression through regulation of beta-site amyloid precursor protein-cleaving enzyme 1. J Neurosci 28 : 1213-1223, 2008. 